University of Nebraska - Lincoln

DigitalCommons@University of Nebraska - Lincoln

Faculty Publications from the Department of Electrical \& Computer Engineering, Department Electrical and Computer Engineering

12-11-1989

\title{
Enhanced magneto-optic Kerr effects in thin magnetic/metallic layered structures
}

\author{
William A. McGahan \\ University of Nebraska-Lincoln \\ Liang-Yao Chen \\ University of Nebraska-Lincoln \\ Z. S. Shan \\ University of Nebraska-Lincoln \\ David J. Sellmyer \\ University of Nebraska-Lincoln, dsellmyer@unl.edu \\ John A. Woollam \\ University of Nebraska-Lincoln, jwoollam1@unl.edu
}

Follow this and additional works at: https://digitalcommons.unl.edu/electricalengineeringfacpub

Part of the Electrical and Computer Engineering Commons

McGahan, William A.; Chen, Liang-Yao; Shan, Z. S.; Sellmyer, David J.; and Woollam, John A., "Enhanced magneto-optic Kerr effects in thin magnetic/metallic layered structures" (1989). Faculty Publications from the Department of Electrical and Computer Engineering. 44.

https://digitalcommons.unl.edu/electricalengineeringfacpub/44

This Article is brought to you for free and open access by the Electrical \& Computer Engineering, Department of at DigitalCommons@University of Nebraska - Lincoln. It has been accepted for inclusion in Faculty Publications from the Department of Electrical and Computer Engineering by an authorized administrator of DigitalCommons@University of Nebraska - Lincoln. 


\title{
Enhanced magneto-optic Kerr effects in thin magnetic/metallic layered structures
}

\author{
William A. McGahan, Liang-Yao Chen, Z. S. Shan, D. J. Sellmyer, and John A. Woollam \\ Departments of Electrical Engineering and Physics, University of Nebraska, Lincoln, Nebraska 68588-0511
}

\begin{abstract}
Enhanced Kerr rotation spectra are measured in thin magnetic layers on silver. Also, variable angle of incidence spectroscopic ellipsometry is employed to measure the optical dielectric function of both the thin magnetic layer and the underlying thick silver layer. These results are explained quantitatively using the electromagnetic theory for reflection of light from multiple layers of isotropic and gyrotropic materials.
\end{abstract}

Applied Physics Letters is copyrighted by The American Institute of Physics.

DOI: $\quad 10.1063 / 1.102004$

Permalink: $\quad$ http://link.aip.org/link/?APPLAB/55/2479/1 


\title{
Enhanced magneto-optic Ker effects in thin magnetic/metallic layered structures
}

\author{
William A. McGanan, Liang-Yao Chen, Z.S. Shan, D. J. Sellmyer, and John A. Woollam \\ Departments of Electrical Eingineering and Physics, University of Nebraska, Lincoh, Nebraska 68588-0511
}

(Received 20 September 1989; accepted for publication 12 October 1989)

\begin{abstract}
Enhanced Kerr rotation spectra are measured in thin magnetic layers on silver. Aiso, variable angle of incidence spectroscopic ellipsometry is employed to measure the optical dielectric function of both the thin magnetic layer and the underlying thick silver layer. These results are explained guantitatively using the electromagnetic theory for reflection of light from multiple layers of isotropic and gyrotropic materials.
\end{abstract}

In 1987, Feil and Haas found that spectrally sharp magneto-optic Kerr effects (MOKEs) exhibited by certain materials could be explained as a consequence of classical plasma resonance of charge carriers. 'A number of subsequent attempts to enhance the MOKE by alloying or layering mag. netic materials with nonmagnetic metals possessing sharp plasma edges have enjoyed various degrees of success. Katayama et al., for example, have presented MOKE data on $\mathrm{Fe} / \mathrm{Cu}$ multilayers showing enhanced rotations at photon energies corresponding to the plasma edge of copper. ${ }^{2}$

Schoenes and Reim ${ }^{3}$ and Feil and Haas ${ }^{4}$ further discussed the interpretation of MOKE data in similar systems. Recently, Reim and Weller presented MOKE data on thin TbFeCo deposited on copper and siver in which they demonstrate enhancement of the Kerr rotation of up to factors of 2 (copper underlayer) to 5 (silver underlayer) over the bulk values. These enhancements occur at photon energies which are significantly removed from the plasma edge of the underlayer, but at which the optical constants $n$ and $k$ of the substrate are smail. ${ }^{5}$ Thus, there exists growing evidence that enhanced MOKEs can occur in layered structures exhibiting unusual optical dielectric functions.

The purpose of this letter is twofold. First, we present experimental data showing large resonant MOKE in magnetic multilayers deposited on silver, with enhancements over bulk values of up to a factor of 4. Second, we use the experimental data in conjunction with the electromagnetic model to demonstrate the fundamental dependence of the MOKE in these structures on $n$ and $k$ and, most important, the reffectance of the structure. In this way we can completely understand the spectral MOKE behavior in these structures, and can engineer the MOKE spectra as desired through adjustments in layer thickness and choice of substrate material.

The samples used for these experiments consisted of glass substrates on which $500-\AA$-thick silver films were evaporated, followed by magnetron-sputtered, compositionally modulated $\mathrm{Dy}(7 a) / \mathrm{Co}(5 \mathrm{~A})$ magnetic layers. The various samples had amorphous magnetic layers of total nominal thicknesses of $72,108,204,372$, and $2000 \AA$. From both the MOKE hysteresis loops and independently measured magnetization measurements, we know that these films exhibit perpendicular magnetic anisotropy.

The samples were characterized by normal incidence Kerr spectroscopy (NIKS), which provides the Kerr rotation at normal incidence, and variable angle spectroscopic ellinsometry (VASE), which yields the pseudodielectric function for the structure. Subsequent anaiysis of the VASE data using a multilayer optical modeling program ${ }^{7.8}$ provided the optical constants for both the silver layer and the magnetic overlayers. Bulk values for both the optical and magneto-optical constants were obtained from the $2000-\AA$ thick sample using a VASE system adapted to make MOKE measurements."

Figure 1 shows the measured MOKE spectra over the photon energy range $1.5-3.7 \mathrm{eV}$. Note that the position and magnitude of the rotation peak is extremely sensitive to the overlayer thickness, and that the peak broatens and shifts to iower energies as the overlayer becomes thicker.

Figure 2 shows the reflectivity spectra for the same set of samples. These spectra are derived from the VASE measurements previously described. Note the change in the character of the spectra from silver-like to the smooth bulk Dy/Co curve as the overlayer thickness is increased. Also note that, as would be expected, the energies at which the MOKE peaks occur in Fig. 1 coincide with the energies at which the reflectance is minima in Fig. 2. This is due to the cancella tion of the isotropic component of the reflected beam associated with the refiectance minima in Fig. 2.

Figure 3 shows the real $(E 1)$ and imaginary $(E 2)$ parts of the complex optical dielectric function $\epsilon_{x x}$ for the Dy/Co layers for all samples, obtained from analysis of VASE data. Notice that there is a distinct dependence on the overlayer thickness. The off-diagonal elements of the dielectric tensor

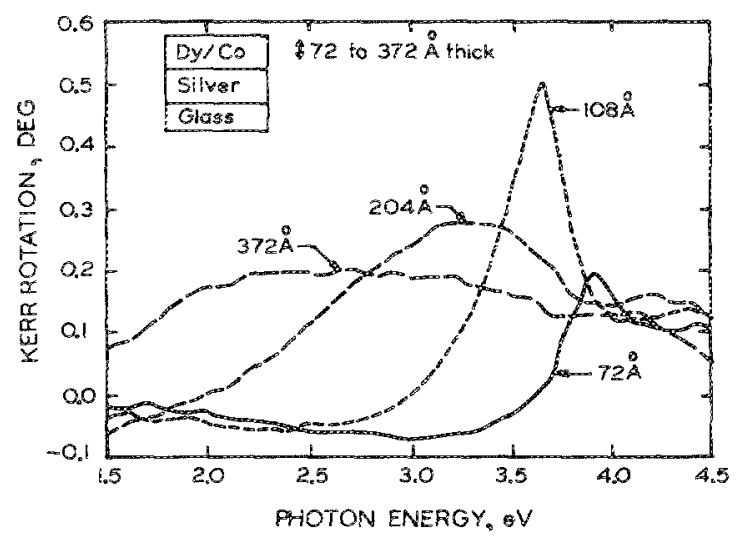

FIG. 1. Measured magneto-optic Kerr rotations in a series of Dy $(7 \mathrm{~A})$ $\mathrm{Co}(5 \mathrm{~A}) / \mathrm{Ag} / \mathrm{glass}$ samples, with the Dy/Co multiayer total thicknesses indicated. 


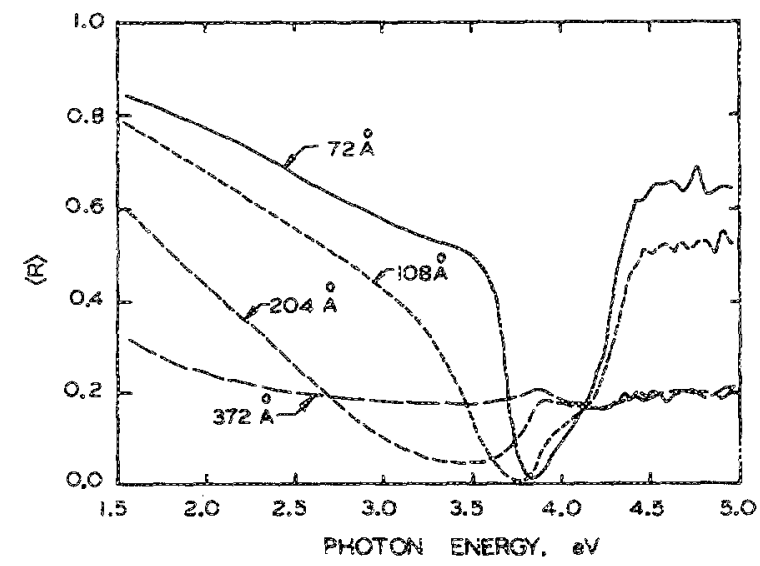

FIG. 2. Refectivity spectra for samples deseribed in Fig. 1.

$\left(\varepsilon_{x y}\right)$ were obtained for the thick $(2000 \AA)$ sample from variable angle spectroscopic magneto-optic ellipsometry. ${ }^{9}$

Using the values for the complete diclectric tensor for each layer in the system, we calculate the expected Kerr rotation spectra for the samples. For this calculation we use the bulk optical and magneto-optical constants for the layers, i.e., we assume that the optical and magneto-optical constants remain relatively constant over the range of layer thicknesses in the study. This calculation is performed using the characteristic matrix method, where a transfer matrix is calculated to express the relationship between the tangential field components at the front and back sides of a given film. ${ }^{10}$ After the characteristic matrix has been calculated for each

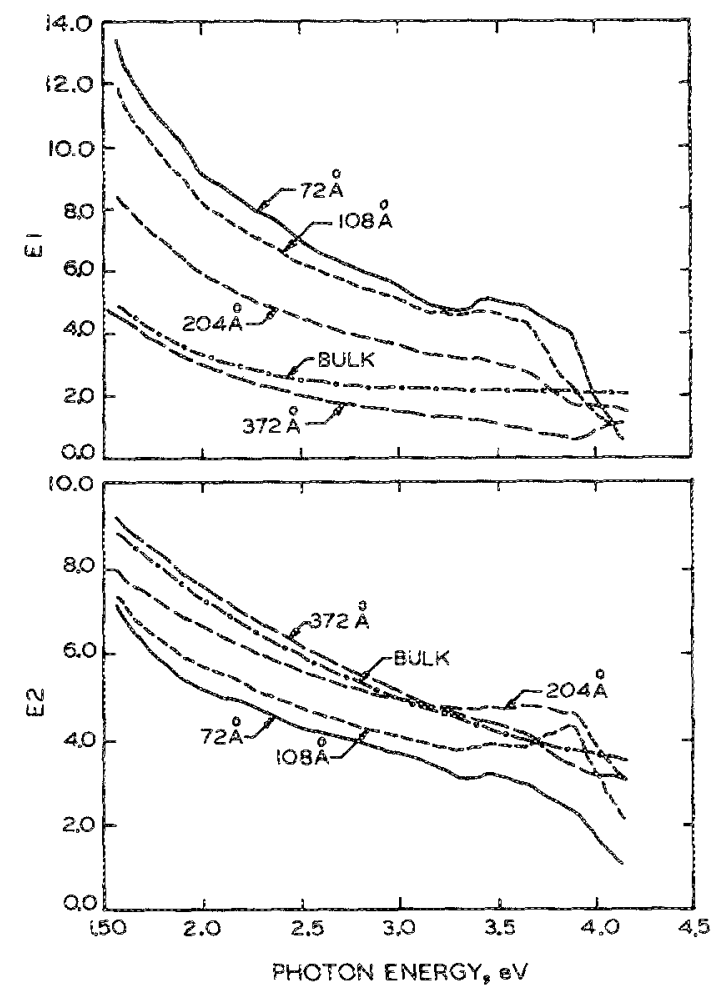

FIG. 3. Measured real $\left(E_{1}\right)$ and imaginary $\left(E_{2}\right)$ parts of the onticat dielectric response furrction for the $D y(7 A) / \operatorname{Co}(S A)$ layers with the total thicknesses indicated.

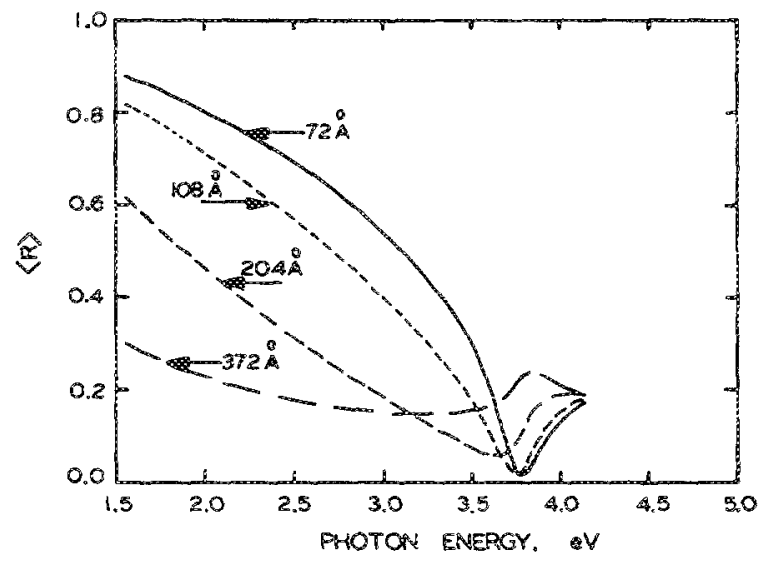

FIG. 4. Caleulated reflectance for the $\operatorname{Dy}(7 A) / C o(5 A)$ layers.

layer, elementary matrix manipulations yield both the opticai and magneto-opticai spectra for the system of interest. Results of these calculations for the samples previously described are shown in Figs. 4 and 5 . Note in Fig. 5 that the peak rotation value is very narrow and highly sensitive to the thickness of the overlayer. For this reason, we expect qualitative agreement between measured and calculated spectra, as sight errors in overlayer thickness will greatly effect the calculated spectra. We plan to alleviate this problem by implementing model firting algorithms to perforn a best-fit analysis of the experimental data.

The resonant behavior of both the observed and calculated spectra is quite easily understood in terns of the optical constants of the system. As the overiayer thickness goes from very thin to very thick, the optical character of the sysiem changes smoothly from that of the substrate (silver in this case) to that of the overlayer. This effect is most easily observed in the refiectance spectra (Fig. 2). Note that the

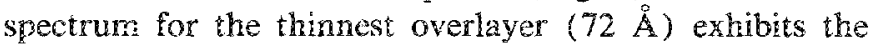
sharp plasma edge and reflectance minimum characteristic of the silver underlayer, while the thickest sample shows the structureless behavior of the amorphous overlayer. This smooth mixing of the optical character of the system is the sole cause of the resonant behavior of the Kerr spectra. For the thin overlayers, the isotropic component of the reffected beam is suppressed at the plasma edge of the silver under-

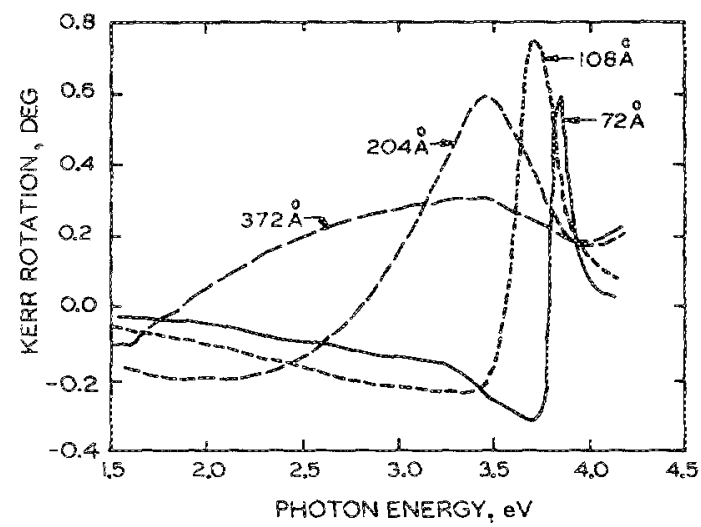

WG. 5. Calculated Kerr rotation as a function of Dy/Co layer thickness and photon energy for the structure shown in the inset of Fig. 1. 
layer. As a basic tool for understanding, we recall the basic equation for the Kerr effect in a system described by a single Jones matrix:

$$
E_{r}=\left(\begin{array}{ll}
R_{p} & K_{p} \\
K_{s} & R_{s}
\end{array}\right) E_{i}
$$

At normal incidence, the complex Kerr effect is given by $u_{K}=\theta_{k}-i \xi_{k}=K_{p} / R_{s}$. Now, $K_{p}$ represents the anisotropic component of the scattered light, while $R_{s}$ will represent the isotropic component. We see that, in this approximation, minimization of the isotropic reffected signal will provide enhancement of the derived parameter which is the Kerr cffect. Hence, a system of layers designed to suppress the isotropic component of the reflected light will display an enhanced Kerr effect, provided there exists some anisotropically scattered component of the beam.

In conclusion, we have offered a simple explanation for the resonant enhancement of the polar Kerr effect in layered systems such as the magnetic layer/silver layer system inves- tigated in this work. We have shown that this enhancement is controllable due to the smooth dependence on the overlayer thickness, and that it is entirely a consequence of the optical character of the system.

'B. Feil and C. Has, Phys. Rev. Let, 58, 65 (1987).

${ }^{2}$ T. Katayama, Y. Suzuki, H. Awano, Y. Mishihara, and N. Koshizuka, Phys. Rev. Lett. 60, 1426 (1988).

${ }^{3}$. Schoenes and W. Reim, Phys. Rev. Lett. 60, 1988 (1988).

${ }^{4}$ H. Feil and C. Hass, Phys. Rev. Lett, 60, 1989 (1988)

${ }^{5}$ W. Keim and D. Weller, Appl. Phys. Lett. 53, 2453 (1988).

TZ. S. Shan and D. J. Sellmyer, J. Appl. Phys. 4745 (1988)

7J. A. Woollam, P. G. Snyder, and M. C. Rost, Thin Solid Films 166,317 (1988).

${ }^{8}$. A. Woollam, P. G. Snyder, and M. C. Rost, Mater. Res. Soc. Symp. Proc. 93, 203 (1987)

'W. A. McGahan, Z. S. Shan, and J. A. Woollam, Appt. Phys. Commun. \&, 209 (1988).

10W. A. McGahan and J. A. Woollam, Appl. Phys. Commun. 8, 1 (1989). 